\title{
Kinetic Resolution of Diols and Pyridyl Alcohols by $\mathrm{Cu}(\mathrm{II})$ (borabox)- Catalyzed Acylation
}

\author{
Clément Mazet, Stephen Roseblade, Valentin Köhler and Andreas Pfaltz*
}

\section{Supporting Information}

All reactions were carried out under an inert atmosphere of argon or nitrogen, unless otherwise noted. Solvents were distilled according to standard procedures. NMR spectra were recorded on 400 and 500 Bruker Avance spectrometers. Infrared spectra were obtained on Shimadzu FTIR-8400S spectrometer using neat samples. Optical rotations were measured on a Perkin Elmer polarimeter 341 equipped with a Na-lamp. Elemental analyses were obtained from the Micro-Analytical Laboratory of the Department of Chemistry of the University of Basel.

General procedure for the synthesis of the lithium salts (3)-Li and the ligands 3a-e. ${ }^{1}$ $t$-BuLi $(1.7 \mathrm{M}$ in hexanes, $1.78 \mathrm{~mL}, 3.03 \mathrm{mmol})$ was added at $-78{ }^{\circ} \mathrm{C}$ over a 10 minutes period to a solution of $2 \mathrm{H}$-oxazoline $(2.75 \mathrm{mmol})$ in $100 \mathrm{~mL}$ of $\mathrm{THF}$, resulting in a pale yellow solution. After stirring for 30 minutes at this temperature, a solution of $\mathrm{R}_{2} \mathrm{BCl}$ $(1.38 \mathrm{mmol})$ in toluene $(5 \mathrm{~mL})$ was added through a cannula and the cooling bath immediately removed after addition was complete. After 4 to $12 \mathrm{~h}$, the reaction was complete and the volatile compounds were removed under vacuum. The remaining foamy residue was redissolved in benzene, filtered to remove $\mathrm{LiCl}$, and the solvent evaporated again. After washing with hexanes $(3 \times 20 \mathrm{~mL})$ and drying, the ligand salt was isolated as a highly hygroscopic white solid.

General procedure for the conversion of the lithium salts into their protonated forms. ${ }^{1}$ The lithium salt of the Bora-BOX (3)-Li was dissolved in a minimum amount of the eluent mixture (typically, hexanes : ethyl acetate : $\mathrm{Et}_{3} \mathrm{~N}=10: 1: 0.5$ ) and directly transferred to a silica gel column $(12 \mathrm{~cm} ; \varnothing=2 \mathrm{~cm})$. After elution and evaporation of the solvents, the product was isolated in its protonated form.

General procedure for the kinetic resolution of 1,2-diols and pyridyl alcohols. Ligand $(1.1 \mathrm{~mol} \%)$ and $\mathrm{CuCl}_{2}(1.0 \mathrm{~mol} \%)$ were dissolved in $2.5 \mathrm{~mL}$ of dichloromethane and the resultant solution was stirred at room temperature for 2-3 hours before the appropriate alcohol $(1.0 \mathrm{mmol})$ was added at room temperature. After cooling the reaction mixture to $0{ }^{\circ} \mathrm{C}, 86 \mu \mathrm{l}$ of $N, N$-diisopropylethylamine $(1.0 \mathrm{mmol})$ and $29 \mu \mathrm{L}$ of benzoylchloride $(0.51 \mathrm{mmol})$ were successively added. The reaction was stirred for an additional $2 \mathrm{~h}$ at $0^{\circ} \mathrm{C}$ and then concentrated to afford the crude product. Flash chromatography using the appropriate eluting mixture allowed separation of the benzoylated product and the enantiomerically enriched starting material. The enantiomeric excess of both materials was determined by HPLC or GC. The diol:benzoylated product ratio established by ${ }^{1} \mathrm{H}$ NMR spectroscopy, before column chromatography, compared well to the calculated $C$ value in all cases $\left(\mathrm{C}_{\text {calc. }}=\right.$ ee $_{\text {alcohol }} /$ $\left(e_{\text {alcohol }}+\right.$ ee $\left.\left._{\text {benzoylated product }}\right)\right){ }^{2}$ Spectroscopic analysis of benzoylated products arising from diols were in good agreement with the published data. ${ }^{3}$ 
Screening of the catalyst loading:
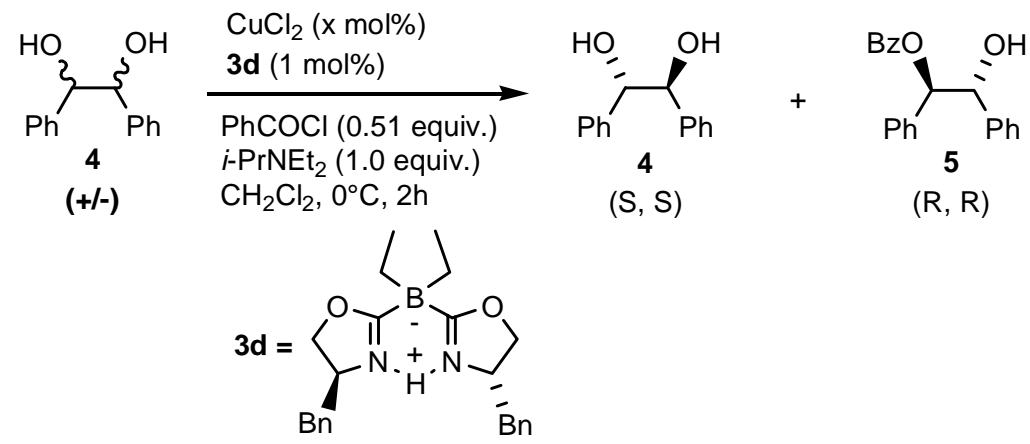

\begin{tabular}{cccccc}
\hline Run & x mol \% & ee $\mathbf{5}(\%)^{\mathrm{a}}$ & ee $\mathbf{4}(\%)^{\mathrm{a}}$ & $\mathrm{C}(\%)^{\mathrm{b}}$ & $\mathrm{S}^{\mathrm{b}}$ \\
\hline 1 & 5.0 & 93 & 86 & 48 & 76 \\
2 & 2.5 & 93 & 86 & 48 & 76 \\
3 & 1.0 & 93 & 82 & 47 & 71
\end{tabular}

${ }^{\mathrm{a}}$ HPLC assay. ${ }^{\mathrm{b}}$ Reference 2.

Throughout this study conversion $(C)$ and enantioselectivity $(S)$ were determined using ee values obtained from chiral HPLC of the product (pr) and recovered starting material $(\mathrm{sm}) ; \mathrm{C}=\mathrm{ee}_{\mathrm{sm}} /\left(\mathrm{ee}_{\mathrm{sm}}+\mathrm{ee}_{\mathrm{pr}}\right) . S=\ln \left[1-\mathrm{C}\left(1+\mathrm{ee}_{\mathrm{pr}}\right)\right] / \ln \left[1-\mathrm{C}\left(1-\mathrm{ee}_{\mathrm{pr}}\right)\right]=\ln \left[(1-\mathrm{C})\left(1-\mathrm{ee}_{\mathrm{sm}}\right)\right] / \ln [(1-$ C) $\left.\left(1+e_{s m}\right)\right]$. See reference 2 for details.

\section{Kinetic resolution of 1,2-phenylethanediol:}

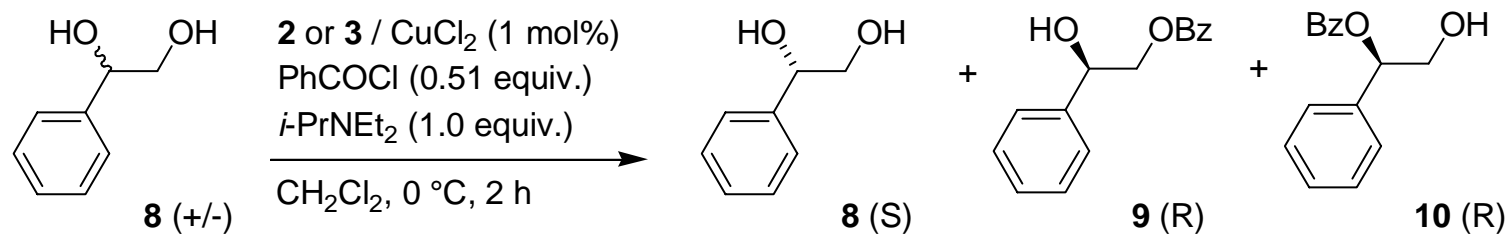

\begin{tabular}{ccccccccc}
\hline ligand & $\mathrm{ee} \mathbf{8}(\%)^{a}$ & $\mathrm{ee} \mathbf{9}(\%)^{a}$ & $\mathrm{ee10}(\%)^{a}$ & $\mathrm{rr}^{b}$ & $C(\%)^{c}$ & $\alpha_{7}{ }^{d}$ & $a_{\mathbf{8}}{ }^{d}$ & $S^{c}$ \\
\hline 2b & 21 & 44 & 88 & $3.8: 1$ & 28 & 1.6 & 25 & 4.1 \\
3d & 45 & 48 & 95 & $5: 1$ & 45 & 1.3 & 148 & 5.3 \\
3e & 42 & 48 & 84 & $1.8: 1$ & 47 & 0.5 & 22.2 & 4.2
\end{tabular}

${ }^{a}$ HPLC assay. ${ }^{b} \mathrm{rr}=[\mathbf{9}] /[\mathbf{1 0}]$; determined by NMR. ${ }^{c} C=(1+\mathrm{rr}) \mathrm{ee} 8 /\left[\operatorname{rr}\left(\right.\right.$ ee8-ee9)+ee8-ee10]. ${ }^{d} \alpha_{7}=\operatorname{rr}[(1-$ ee9)/(1+ee10)]. ${ }^{e} \alpha_{8}=\operatorname{rr}[(1+\mathrm{ee} 9) /(1-\mathrm{ee} \mathbf{1 0})] .{ }^{f} S=\ln [1-\mathrm{C}(1+\mathrm{ee} \mathbf{9})] / \ln [1-\mathrm{C}(1-\mathrm{ee} \mathbf{9})]$; see Reference 2. 
Pyridyl Alcohol Synthesis: In general a fused, bicyclic pyridine was converted to the corresponding $\mathrm{N}$-oxide using $m \mathrm{CPBA}$ (1.1 equiv., $\mathrm{CH}_{2} \mathrm{Cl}_{2}, \mathrm{rt}, 16 \mathrm{~h}$ ). The crude $\mathrm{N}$-oxide was then acetylated using trifluoroacetic anhydride (2.5 equiv., $\mathrm{CH}_{2} \mathrm{Cl}_{2}, 0{ }^{\circ} \mathrm{C}$ to $\mathrm{rt}, 16 \mathrm{~h}$ ) and subsequently treated with aqueous lithium hydroxide $\left(2 \mathrm{~N} \mathrm{LiOH} / \mathrm{CH}_{2} \mathrm{Cl}_{2}, \mathrm{rt}, 16 \mathrm{~h}\right)$ to give the pyridyl alcohol after purification by column chromatography or recrystallisation. ${ }^{4}$

General Procedure for Synthesis of Racemic Benzoates: Anhydrous copper (II) chloride (1.3 mg, $4 \mathrm{~mol} \%$ ) and 2,2'-bipyridyl (1.6 mg, $4 \mathrm{~mol} \%$ ) were stirred together in dry dichloromethane $(1 \mathrm{~mL})$ for 2 hours, under argon, to give a green solution. The pyridyl alcohol $(0.25 \mathrm{mmol})$ was then added and the resulting solution was cooled to $0{ }^{\circ} \mathrm{C}$ in an ice bath. $N, N$-Diisopropylethylamine $(43 \mu \mathrm{L}, 0.25 \mathrm{mmol})$ and benzoyl chloride ( 29 $\mu \mathrm{L}, 0.25 \mathrm{mmol}$ ) were then added, in that order, and the reaction mixture was stirred for at least two hours at room temperature. The reaction mixture was diluted with dichloromethane $(2 \mathrm{~mL})$ and water $(2 \mathrm{~mL})$ was then added; the organic layer was separated and the aqueous layer extracted once with dichloromethane $(2 \mathrm{~mL})$. The organic layer was dried $\left(\mathrm{MgSO}_{4}\right)$, filtered and concentrated to give the crude benzoate which was purified by column chromatography.

\section{7-Benzoxy-6,7-dihydro-1,5-pyridine 16}

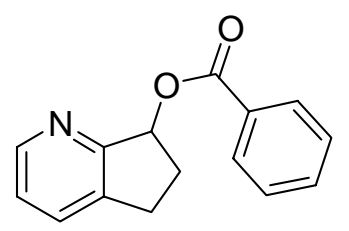

7-Hydroxy-6,7-dihydro-1,5-pyridine ${ }^{5}$ was treated as described above to give the corresponding benzoate as a colorless oil which solidified on standing. $\mathrm{R}_{\mathrm{f}} 0.38$ (2:1 hexanes:EtOAc); ${ }^{1} \mathrm{H}$ NMR: (500 MHz, $\left.\mathrm{CDCl}_{3}\right): 8.51(1 \mathrm{H}, \mathrm{d}, J 4.7, \mathrm{NCH}), 8.04(2 \mathrm{H}, \mathrm{d}, J$ 7.1, 2 x C=OCCH), $7.61(1 \mathrm{H}, \mathrm{d}, J 7.2), 7.50(1 \mathrm{H}, \mathrm{t}, J 10.0), 7.37(2 \mathrm{H}, \mathrm{t}, J 7.9), 7.19(1 \mathrm{H}$, dd, $J$ 7.7, 3.1), 6.38 (1H, dd, $J$ 7.4, 4.7, CHO), $3.12\left(1 \mathrm{H}, \mathrm{ddd}, J 16.1,8.8,5.2, \mathrm{ArCH}_{a} \mathrm{H}_{\mathrm{b}}\right.$ ), 2.90-2.96 $\left(1 \mathrm{H}, \mathrm{m}, \mathrm{ArCH}_{\mathrm{a}} H_{b}\right), 2.68-2.75(1 \mathrm{H}, \mathrm{m}), 2.18-2.24(1 \mathrm{H}, \mathrm{m}) ;{ }^{13} \mathrm{C}$ NMR: $(125$ $\left.\mathrm{MHz}, \mathrm{CDCl}_{3}\right)$ : $166.8(\mathrm{C}=\mathrm{O}), 161.0,149.3,138.0,133.5,133.3,130.7,130.3,128.6$, 123.8, 78.1, 31.2, 28.3; MS (FAB) $\mathrm{m} / \mathrm{z}$ (rel. intensity): $240\left(\mathrm{M}+\mathrm{H}^{+}, 85\right)$. Elemental analysis calcd (\%) for $\mathrm{C}_{15} \mathrm{H}_{13} \mathrm{NO}_{2}$ (239.27): C 75.30, $\mathrm{H} 5.48, \mathrm{~N} 5.85$; found: $\mathrm{C} 75.12, \mathrm{H}$ $5.48, \mathrm{~N} 5.89$. 


\section{2-Phenyl-7-benzoxy-6,7-dihydro-1,5-pyridine 17}

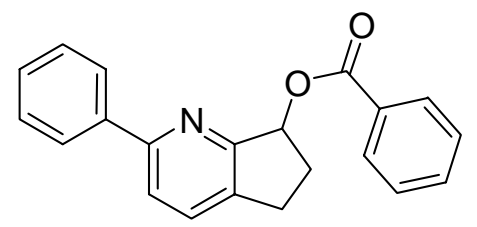

2-Phenyl-7-hydroxy-6,7-dihydro-1,5-pyridine ${ }^{6}$ was treated as described above to give the corresponding benzoate as white needles. M.p. 138-139 ${ }^{\circ} \mathrm{C} ; \mathrm{R}_{\mathrm{f}} 0.32\left(1: 1 \mathrm{CH}_{2} \mathrm{Cl}_{2}\right.$ :hexane); ${ }^{1} \mathrm{H}$ NMR: (500 MHz, $\left.\mathrm{CDCl}_{3}\right) 8.07(2 \mathrm{H}, \mathrm{m}), 7.99(2 \mathrm{H}, \mathrm{m}), 7.66-7.71(2 \mathrm{H}, \mathrm{m}), 7.54(1 \mathrm{H}, \mathrm{t}$, $J$ 7.4), 7.37-7.47 (5H, m), 6.44 (1H, dd, J 7.1, 4.3, CHO), 3.14-3.21 (1H, m), 2.94-3.01 $(1 \mathrm{H}, \mathrm{m}), 2.73-2.80\left(1 \mathrm{H}, \mathrm{m}, \mathrm{C}=\mathrm{CC} H_{a} \mathrm{H}_{\mathrm{b}}\right), 2.28\left(1 \mathrm{H}, \mathrm{ddd}, J 18.3,8.9,4.4, \mathrm{C}=\mathrm{CCH}_{\mathrm{a}} H_{b}\right) ;{ }^{13} \mathrm{C}$ NMR: (125 MHz, $\left.\mathrm{CDCl}_{3}\right) 166.5(\mathrm{C}=\mathrm{O}), 160.7,157.2,139.4,136.2,133.6,132.9,130.5$, 129.8, 128.8, 128.7, 128.3, 127.0, 120.6, 78.1 ( $\left.\mathrm{CH}_{2} \mathrm{O}\right), 31.2,27.8$; MS (FAB) $m / z$ (rel. intensity): $316\left(\mathrm{M}+\mathrm{H}^{+}, 50\right)$. Elemental analysis calcd (\%) for $\mathrm{C}_{21} \mathrm{H}_{17} \mathrm{NO}_{2}$ (315.37): $\mathrm{C}$ 79.98, H 5.43, N 4.44; found: C 79.57, H 5.52, N 4.46.

\section{8-Benzoxy-5,6,7,8,-tetrahydroquinoline 18}

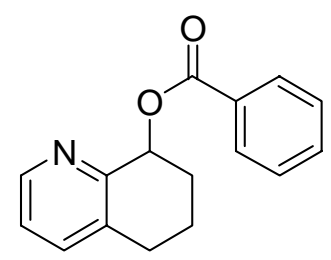

8-Hydroxy-5,6,7,8,-tetrahydroquinoline ${ }^{7}$ was treated as described above to give the corresponding benzoate as a colourless oil which crystallises on standing. $\mathrm{R}_{\mathrm{f}} 0.35(2: 1$ hexanes:EtOAc); ${ }^{1} \mathrm{H}$ NMR (500 MHz, $\left.\mathrm{CDCl}_{3}\right): 8.49(1 \mathrm{H}, \mathrm{d}, J$ 4.0, $\mathrm{NCH}), 8.04(2 \mathrm{H}, \mathrm{dd}, J$ 8.0, 1.4, 2 x C=OCCH), 7.46-7.54 (2H, m), $7.39(2 \mathrm{H}, \mathrm{t}, J 7.9,2 \times \mathrm{C}=\mathrm{OCCHCH}), 7.17$ $(1 \mathrm{H}, \mathrm{dd}, J$ 7.8, 4.0, NCHCH), $6.25(1 \mathrm{H}, \mathrm{t}, J 4.6, \mathrm{CHO}), 2.92(1 \mathrm{H}, \mathrm{dt}, J$ 17.0, 5.1, $\left.\mathrm{ArCH}_{a} \mathrm{H}_{\mathrm{b}}\right), 2.81\left(1 \mathrm{H}, \mathrm{ddd}, J 16.9,9.6,5.7, \mathrm{ArCH}_{\mathrm{a}} H_{b}\right), 2.27-2.33\left(1 \mathrm{H}, \mathrm{m}, \mathrm{OCHCH}_{a} \mathrm{CH}_{\mathrm{b}}\right)$, 2.10-2.17 (1H, m, OCHCH $\left.\mathrm{CH}_{b}\right), 1.99-2.08\left(1 \mathrm{H}, \mathrm{m}, \mathrm{CH}_{2} \mathrm{CH}_{a} \mathrm{H}_{\mathrm{b}} \mathrm{CH}_{2}\right), 1.86-1.98(1 \mathrm{H}, \mathrm{m}$, $\left.\mathrm{CH}_{2} \mathrm{CH}_{\mathrm{a}} \mathrm{H}_{b} \mathrm{CH}_{2}\right)$; ${ }^{13} \mathrm{C} \mathrm{NMR}:\left(125 \mathrm{MHz}, \mathrm{CDCl}_{3}\right) 166.0(\mathrm{C}=\mathrm{O}), 153.4,147.9,137.1,133.6$, 132.8, 130.7, 129.8, 128.2, 123.1, 71.3 (CHO), 29.2, 28.5, 18.5; MS (FAB) m/z (rel. intensity): $254\left(\mathrm{M}+\mathrm{H}^{+}, 89\right)$. Elemental analysis calcd (\%) for $\mathrm{C}_{16} \mathrm{H}_{15} \mathrm{NO}_{2}$ (253.30): $\mathrm{C}$ 75.87, H 5.97, N 5.53; found: C 75.75, H 5.96, N 5.61. 


\section{2-Phenyl-8-benzoxy-5,6,7,8,-tetrahydroquinoline 19}

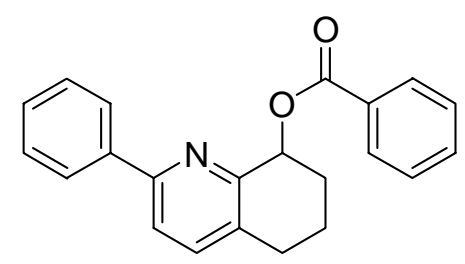

2-Phenyl-8-hydroxy-5,6,7,8,-tetrahydroquinoline ${ }^{8}$ was treated as described above to give the corresponding benzoate as a colorless oil, which solidified slowly on standing. $\mathrm{R}_{\mathrm{f}} 0.26$ (9:1 pentane:EtOAc); ${ }^{1} \mathrm{H}$ NMR: (400 $\left.\mathrm{MHz}, \mathrm{CDCl}_{3}\right) 8.08(2 \mathrm{H}, \mathrm{m}), 7.94-7.97(2 \mathrm{H}, \mathrm{m})$, $7.63(1 \mathrm{H}, \mathrm{d}, J$ 8.1), 7.52-7.56 (2H,m), 7.31-7.44 (5H, m), 6.33 (1H, t, J 5.1, CHO), 2.95 $\left(1 \mathrm{H}, \mathrm{dt}, J 16.9,5.6, \mathrm{ArCH}_{a} \mathrm{H}_{\mathrm{b}}\right), 2.84\left(1 \mathrm{H}, \mathrm{dd}, J 16.9,8.8,5.8, \mathrm{ArCH}_{\mathrm{a}} H_{b}\right), 2.29-2.34(1 \mathrm{H}$, $\mathrm{m}), 2.17-2.25(1 \mathrm{H}, \mathrm{m}), 2.05-2.11(1 \mathrm{H}, \mathrm{m}), 1.89-1.97(1 \mathrm{H}, \mathrm{m}) ;{ }^{13} \mathrm{C}$ NMR: $(100 \mathrm{MHz}$, $\left.\mathrm{CDCl}_{3}\right) 166.6$ (CO), 155.4, 153.6, 139.4, 138.2, 133.1, 132.2, 131.4, 130.2, 129.1, 129.0, 128.7, 127.1, 120.0, 72.2 (CHO), 29.6, 28.6, 19.2; MS (FAB) m/z (rel. intensity): 330 $\left(\mathrm{M}+\mathrm{H}^{+}, 85\right)$. Elemental analysis calcd $(\%)$ for $\mathrm{C}_{22} \mathrm{H}_{19} \mathrm{NO}_{2}(329.40)$ : C 80.22, H 5.81, N 4.25; found: C 80.32, H 5.63, N 4.27.

\section{2-Chloro-8-benzoxy-5,6,7,8,-tetrahydroquinoline 20}

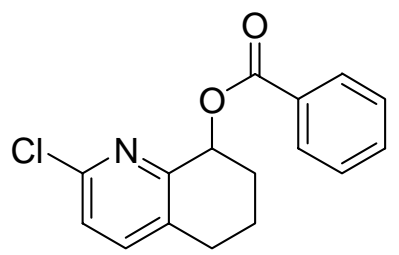

2-Chloro-8-hydroxy-5,6,7,8,-tetrahydroquinoline ${ }^{9}$ was treated as described above to give the corresponding benzoate as white needles. M. p. $118-119{ }^{\circ} \mathrm{C} ; \mathrm{R}_{\mathrm{f}} 0.2(9: 1$ pentane:EtOAc); ${ }^{1} \mathrm{H}$ NMR: (400 MHz, $\left.\mathrm{CDCl}_{3}\right): 8.00-8.04(2 \mathrm{H}, \mathrm{m}), 7.53(1 \mathrm{H}, \mathrm{tt}, J 6.8$, 1.2, $\mathrm{CHCHCHCCO} 2), 7.38-7.46(3 \mathrm{H}, \mathrm{m}), 7.21(1 \mathrm{H}, \mathrm{d}, J 8.1), 6.12(1 \mathrm{H}, \mathrm{t}, J 4.3), 2.90$ $\left(1 \mathrm{H}, \mathrm{dt}, J 16.9,4.8, \mathrm{ArCH}_{a} \mathrm{H}_{\mathrm{b}}\right), 2.77\left(1 \mathrm{H}, \mathrm{ddd}, J 15.4,9.6,5.8, \mathrm{ArCH}_{\mathrm{a}} H_{b}\right), 2.27-2.35(1 \mathrm{H}$, $\mathrm{m}), 1.94-2.13(3 \mathrm{H}, \mathrm{m}), 1.83-1.92(1 \mathrm{H}, \mathrm{m}) ;{ }^{13} \mathrm{C} \mathrm{NMR}$ : (100 MHz, $\left.\mathrm{CDCl}_{3}\right) 166.1(\mathrm{C}=\mathrm{O})$, 154.2, 149.3, 140.4, 133.2, 133.0, 131.0, 130.2, 128.6, 124.4, 71.3 (CHO), 29.2, 28.2, 18.5; MS (FAB) $\mathrm{m} / \mathrm{z}$ (rel. intensity): $288\left(\mathrm{M}+\mathrm{H}^{+}, 90\right)$. Elemental analysis calcd (\%) for $\mathrm{C}_{16} \mathrm{H}_{14} \mathrm{NO}_{2} \mathrm{Cl}$ (287.74): C 66.79, $\mathrm{H}$ 4.90, N 4.87; found: C 66.62, H 4.93, N 4.89.

Gram synthesis of both enantiomers of 2-phenyl-8-hydroxy-5,6,7,8,-tetrahydroquinoline:

Anhydrous copper(II) chloride (6.0 mg, $1 \mathrm{~mol} \%)$ and ligand 3d (17.3, mg, 1 mol\%) were dissolved in anhydrous dichloromethane $(5 \mathrm{~mL})$ and stirred for 90 minutes at room temperature under argon. The resulting dark yellow solution was filtered through a syringe filter (CHROMAFIL O-20/15 MS 0.2 $\mu \mathrm{M}$, Macherey-Nagel) into a dry Schlenk tube containing racemic 2-phenyl-8-hydroxy-5,6,7,8,-tetrahydroquinoline 14 (1.0 g, 4.44 mmol) under argon. The resulting green solution was cooled to $0{ }^{\circ} \mathrm{C}$ in an ice bath. $N, N$ - 
diisopropylethylamine (773 $\mu \mathrm{L}, 1$ equiv) was added dropwise, to give a blue/green solution, followed by benzoyl chloride ( $284 \mu \mathrm{L}, 0.55$ equiv). The reaction mixture was stirred overnight, during which time it was allowed to warm slowly to room temperature. Water $(5 \mathrm{~mL})$ was added to the reaction mixture and the organic layer was separated. The aqueous layer was extracted with dichloromethane $(5 \mathrm{~mL})$ and the combined organic layers were dried with $\mathrm{MgSO}_{4}$, filtered and concentrated. The benzoate and alcohol were separated by column chromatography (10:1 pentane:EtOAc) to give the $(R)$-benzoate (694 $\mathrm{mg}, 47 \%, 94 \%$ ee) as a pale green oil $\left(\mathrm{R}_{\mathrm{f}} 0.26,9: 1\right.$ pentane:EtOAc)and the $(S)$ alcohol (426 mg, 43\%, 91\% ee) as a pale green crystalline solid $\left(\mathrm{R}_{\mathrm{f}} 0.13,9: 1\right.$ pentane:EtOAc). The green color seems to be from residual copper which has come through the work-up and column of silica gel. The alcohol was purified further by recrystallisation from hot 1:1 hexane:EtOAc to give $387 \mathrm{mg}$ of $(S)$-alcohol (39\% yield) in 95\% ee as colorless prisms. The literature data for $\mathbf{1 4}$ are not available ${ }^{8}$ and so are reported here; ${ }^{1} \mathrm{H} \mathrm{NMR}\left(400 \mathrm{MHz}, \mathrm{CDCl}_{3}\right): 8.00(2 \mathrm{H}, \mathrm{m}), 7.58(1 \mathrm{H}, \mathrm{d}, J$ 8.0), 7.35-7.50 $(4 \mathrm{H}, \mathrm{m}), 4.75(1 \mathrm{H}, \mathrm{dd}, J$ 9.1, 5.8, CHO $), 4.38(1 \mathrm{H}, \mathrm{br}$ s, OH), 2.80-2.93 (2H, m), 2.33$2.43(1 \mathrm{H}, \mathrm{m}), 1.96-2.10(1 \mathrm{H}, \mathrm{m}), 1.75-1.92(2 \mathrm{H}, \mathrm{m}) ;{ }^{13} \mathrm{C}$ NMR: $\left(100 \mathrm{MHz}, \mathrm{CDCl}_{3}\right) 158.0$, $154.6,139.1,138.3,130.4,129.3,129.1,127.1,119.7,69.5,31.0,28.4,20.1$; MS (EI) $\mathrm{m} / \mathrm{z}$ (rel. intensity) $225\left(\mathrm{M}^{+}, 15\right), 196(30), 169$ (100). Elemental analysis calcd (\%) for $\mathrm{C}_{15} \mathrm{H}_{15} \mathrm{NO}$ (225.29): C 79.97, H 6.71, N 6.22; found: C 79.27, H 6.72, N 6.22; m.p. 88-90 ${ }^{\circ} \mathrm{C} ; \alpha_{\mathrm{D}}+143\left(\mathrm{c}=1\right.$ in $\mathrm{CH}_{2} \mathrm{Cl}_{2}$ ).

Subsequently, the $(R)$-benzoate (330 $\mathrm{mg}, 1 \mathrm{mmol}, 94 \%$ ee) was dissolved in ethanol (20 $\mathrm{mL})$ and $2 \mathrm{~N} \mathrm{NaOH}(1 \mathrm{~mL}, 2 \mathrm{mmol}, 2$ equiv) was added at room temperature. After stirring for 3 hours the reaction was diluted with water $(20 \mathrm{~mL})$ and extracted with dichloromethane $(3 \times 20 \mathrm{~mL})$. The combined organic layers were dried $\left(\mathrm{MgSO}_{4}\right)$, filtered and concentrated to give the crude $(R)$-alcohol as a crystalline solid $(93 \% \mathrm{ee})$. Recrystallisation from hot 1:1 hexane:EtOAc gave $200 \mathrm{mg}(89 \%)$ of the $(R)$-alcohol with 97\% ee. M.p. $85-94{ }^{\circ} \mathrm{C}$.

The absolute configuration of the diols and corresponding benzoates was determined by comparison with literature data. ${ }^{3 a}$ Diols 4 and $\mathbf{6}$ are commercially available in enantiomerically pure form. The stereochemistry of compounds $\mathbf{8 , 9}$ and $\mathbf{1 0}$ was also determined by comparison with literature data. ${ }^{3 a, 10}$

The absolute configuration of pyridyl alcohols 12 and $\mathbf{1 3}$ was assigned by comparison of $\alpha_{D}$ values with those previously reported in the literature. ${ }^{6,7}$

The absolute configuration of the pyridyl alcohols 11, 14 and 15 obtained from kinetic resolution was confirmed as being analogous by single crystal X-ray diffraction studies of the corresponding [iridium $\mathrm{COD}$ ] $\mathrm{BAr}_{\mathrm{F}}$ complexes. ${ }^{4}$ 
Chiral HPLC conditions:

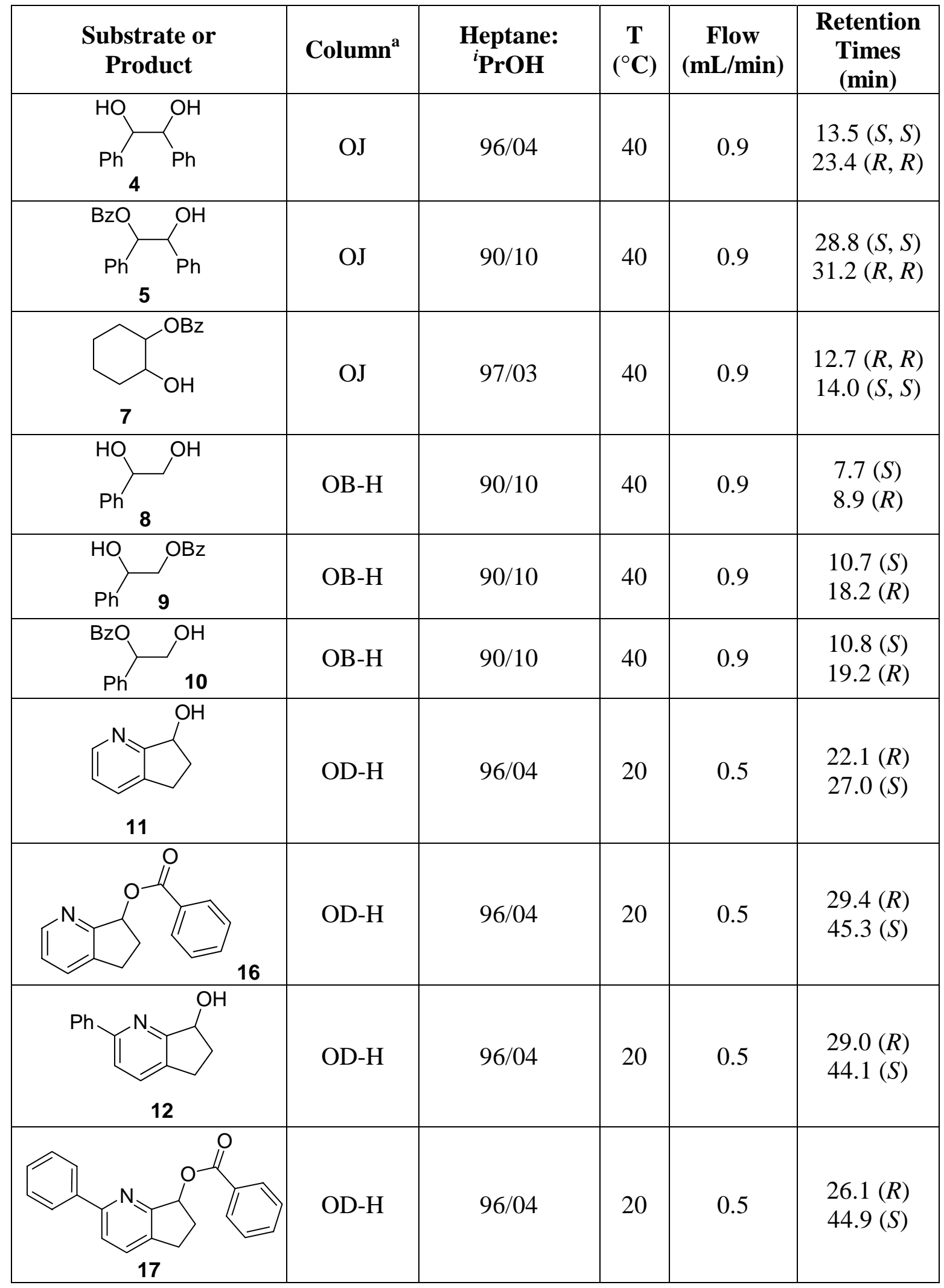




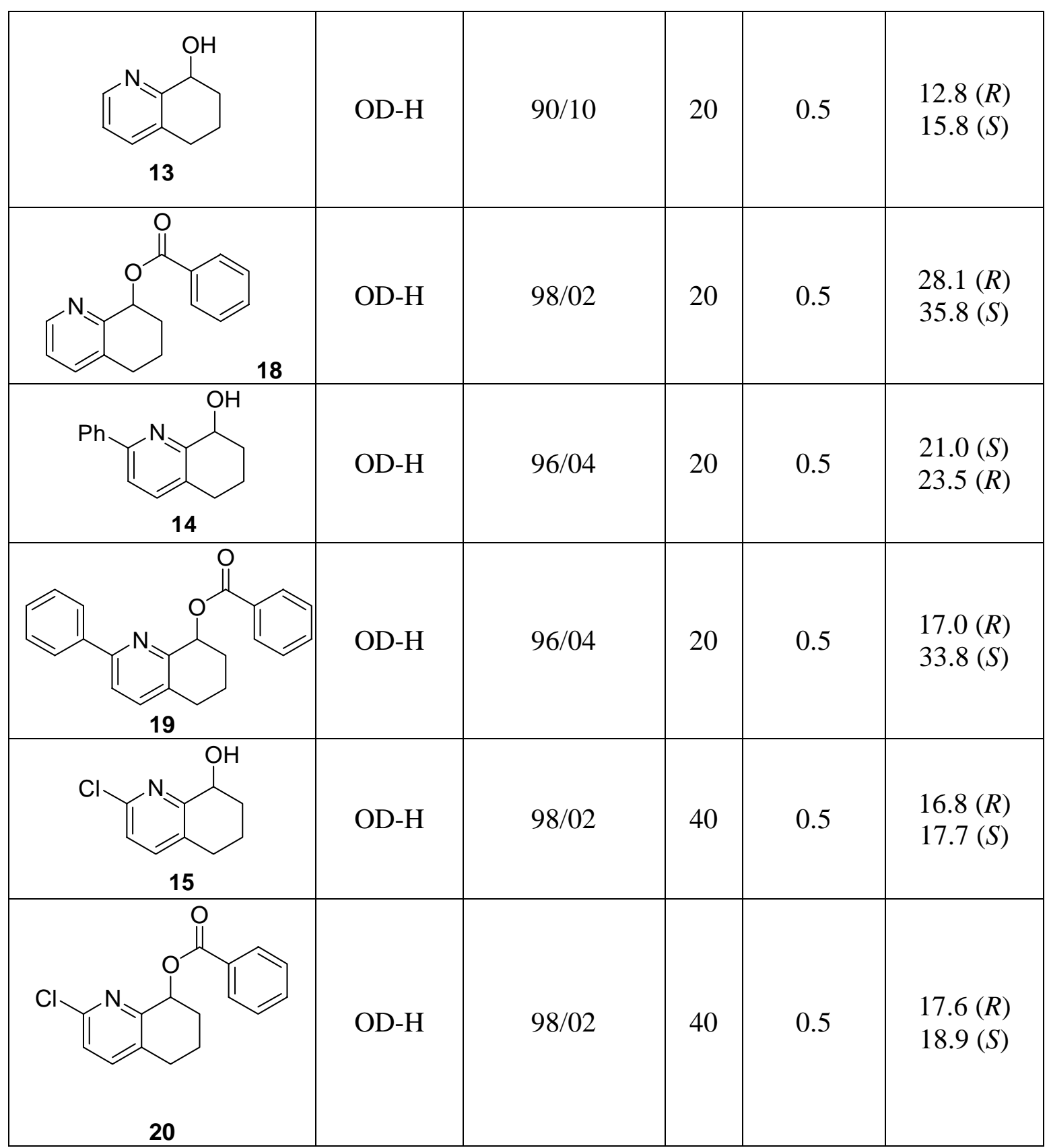

a) Chiralcel columns, $0.46 \mathrm{~cm} \times 25 \mathrm{~cm}$.

GC conditions for trans-1,2-cyclohexanediol 6: Hydroxy- $\beta$-3P-Macherey-Nagel; $25 \mathrm{~m}^{*} 0.25 \mathrm{~mm}$; isothermal $=100{ }^{\circ} \mathrm{C}$; carrier gas $\left(\mathrm{H}_{2}, \mathrm{kPa}\right)=100$ bar. 


\section{References:}

[1] Mazet, C.; Köhler, V.; Pfaltz, A. Angew. Chem., 2005, 117, 4966-4969; Angew. Chem. Int. Ed., 2005, 44, 4488-4891.

[2] a) Kagan, H. B.; Fiaud, J.-C. Top. Stereochem. 1988, 18, 249-330; b) Sih, C. J.; Wu, S. H. Top Stereochem. 1989, 19, 63-125; c) Kagan, H. B. Tetrahedron 2001, 57, 2449-2468; d) Kagan, H. B. Croat. Chem. Acta 1996, 69, 669-680.

[3] a) Iwasaki, F.; Maki, T.; Nakashima, W.; Onomura, O.; Matsumura, Y. Org. Lett., 1999, 1, 969-972; b) Iwasaki, F.; Maki, T.; Onomura, O.; Nakashima, W.; Matsumura, Y. J. Org. Chem., 2000, 65, 996-1002; c) Ciuffreda, P.; Alessandrini, L.; Terraneo, G.; Santaniello, E. Tetrahedron: Asymmetry, 2003, 14, 3197-3201; d) Matsumura, Y.; Maki, T.; Murakami, S.; Onomura, O. J. Am. Chem. Soc., 2003, 125, 2052-2053.

[4] Kaiser, S., PhD Dissertation, University of Basel, 2005.

[5] Robison, M. M. J. Am. Chem. Soc., 1958, 80, 6254-6257.

[6] Kang, J.; Kim, H. Y.; Kim, J. H. Tetrahedron: Asymmetry, 1999, 10, 2523-2533 and references therein.

[7] Uenishi, J.; Hamada, M. Synthesis, 2002, 5, 625-630 and references therein.

[8] Hahn, W. E.; Epsztajn, J. Rocz. Chem., 1963, 37, 403-412.

[9] Zimmerman, S. C.; Zeng, Z.; Wu, W.; Reichert, D. E. J. Am. Chem. Soc., 1991, 113, 183-196.

[10] Brown, J. M.; Murrer, B. A. J. Chem. Soc., Perkin Trans. 2 1982, 489. 